Signal \& Image Processing : An International Journal (SIPIJ) Vol.6, No.1, February 2015

\title{
IMAGE PROCESSING BASED GIRTH MONITORING AND RECORDING SYSTEM FOR RUBBER PLANTATIONS
}

\author{
Chathura Thilakarathne, Padmika Bhanusri, Tharindu Randeny, Harsha \\ Rupasinghe and Chulantha Kulasekere \\ Department of Electronic and Telecommunication Engineering, \\ University of Moratuwa, Moratuwa, Sri Lanka
}

\begin{abstract}
Measuring the girth and continuous monitoring of the increase in girth is one of the most important processes in rubber plantations since identification of girth deficiencies would enable planters to take corrective actions to ensure a good yield from the plantation.

This research paper presents an image processing based girth measurement \& recording system that can replace existing manual process in an efficient and economical manner.

The system uses a digital image of the tree which uses the current number drawn on the tree to identify the tree number \& its width. The image is threshold first \& then filtered out using several filtering criterion to identify possible candidates for numbers. Identified blobs are then fed to the Tesseract OCR for number recognition. Threshold image is then filtered again with different criterion to segment out the black strip drawn on the tree which is then used to calculate the width of the tree using calibration parameters. Once the tree number is identified \& width is calculated the girth the measured girth of the tree is stored in the data base under the identified tree number.

The results obtained from the system indicated significant improvement in efficiency \& economy for main plantations. As future developments we are proposing a standard commercial system for girth measurement using standardized 2D Bar Codes as tree identifiers
\end{abstract}

\section{KEYWORDS}

Image processing, OpenCV, Rubber plantations, Tesseract

\section{INTRODUCTION}

Large scale Rubber plantations are one of the key components of Sri Lankan economic plantation sector and these plantations shall be continuously monitored throughout the year to identify any irregularities as indicators such as degradation of girth may directly correlate with the produced yield. 
Signal \& Image Processing : An International Journal (SIPIJ) Vol.6, No.1, February 2015

One such key parameter is the girth measurement of individual trees and to evaluate the cumulative and integrative girth parametric to identify any diseases, nutrition needs.

The current process for girth measurement is a labor intensive manual process which leads to significant degree of erroneous reporting.

Hence, the requirement for identification of simple technological solution is highly critical and it would benefit in significant cost savings and revenue generations. Aggregated girth measurements are utilized to derive statistical parameters such as Growing Stock, Mean Annual Increment (MAI) and Current Annual Increment (CAI) [1].

\section{LITERATURE SURVEY}

In the Literature Survey, we identify some of the girth measurement techniques used in similar markets.

One such method is the use of IR based distance and angle measurement techniques to identify girth characteristics of vertical cylindrical objects.

The non-contact measurement techniques based on machine vision used in green houses also provides a mechanism to measure the girth of small plants by monitoring the growth of the canopy of the plant. However, this method was not suited for large scale plantations.

Epicom and Perimeter [2] are measuring devices used in Green House horticulture and developed by Wageningen UR. These techniques deploy a mechanical tape measurement around the circumference; hence, even though these approaches provide best accuracy of the girth measurement, there are practical problems in introducing these devices to plantation sector since these approaches tightly follow the existing methodology.

Further products such as TRUE SPAN \& uPhotoMeasure [3] were helpful in developing image processing based system in our design. TRUE SPAN (Vision Based With Measuring System) developed by the automation division of Tata Steel Ltd senses the strip of edges with two very high resolution linear array of CCD cameras used to snap at approximately $20 \mathrm{MHz}$ placed on top of the rolling table. In order to effect the width measurement at the same point with respect to the strop a common clock controller synchronizes the cameras. TRUE SPAN uses triangulation principles to estimate the width.

\section{DESIGN AND IMPLEMENTATION}

System for the measurement of the girth monitoring and recording of a rubber plantation has three distinguished parts. The main and the most challenging part is image processing algorithm which determines the girth increment of the rubber tree. Second part is the recording of the measured values in a database which can be used to give back meaningful data for analysis. Third part is the selection of optimum hardware specifications and developing the design to suit plantation work environments. 


\section{A. Image Processing Subsystem}

The core area of the research was based on image processing subsystem which mainly focused on three key functions;

1. Identifying / Detecting Location of Numbers

2. Number Recognition

3. Width measurement

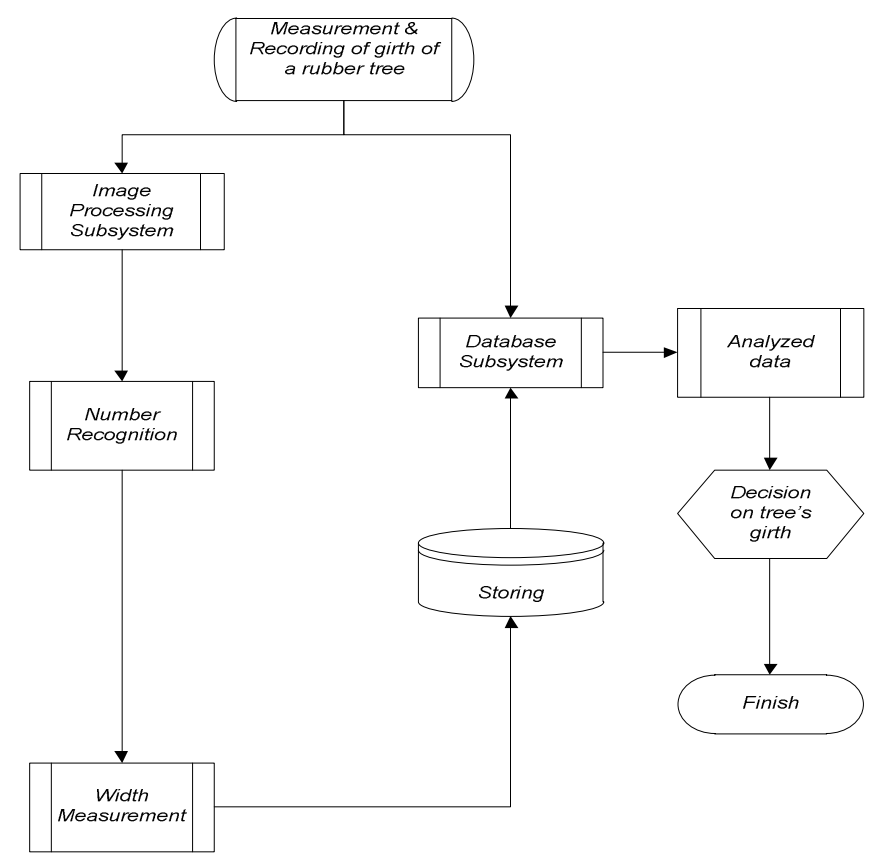

Fig. 1: Image Processing System Architecture

The tools used in image processing sub system consisted of Microsoft Visual Studio 2008 32-bit version with $\mathrm{C}++$ as the high level language. Open Source Computer Vision library better known as OpenCV version 2.1.0 2008 [2, 3], 32 bit version was used as the Image Processing platform. The final system is platform independent since it has not used any Windows proprietary library, even though it was developed in the Windows environment.

\section{Identifying / Detecting Location of Numbers}

Identification and detection of locale of numbers involves several intermediate steps supported by OpenCV algorithms as mentioned in the figure 2. 
Signal \& Image Processing : An International Journal (SIPIJ) Vol.6, No.1, February 2015

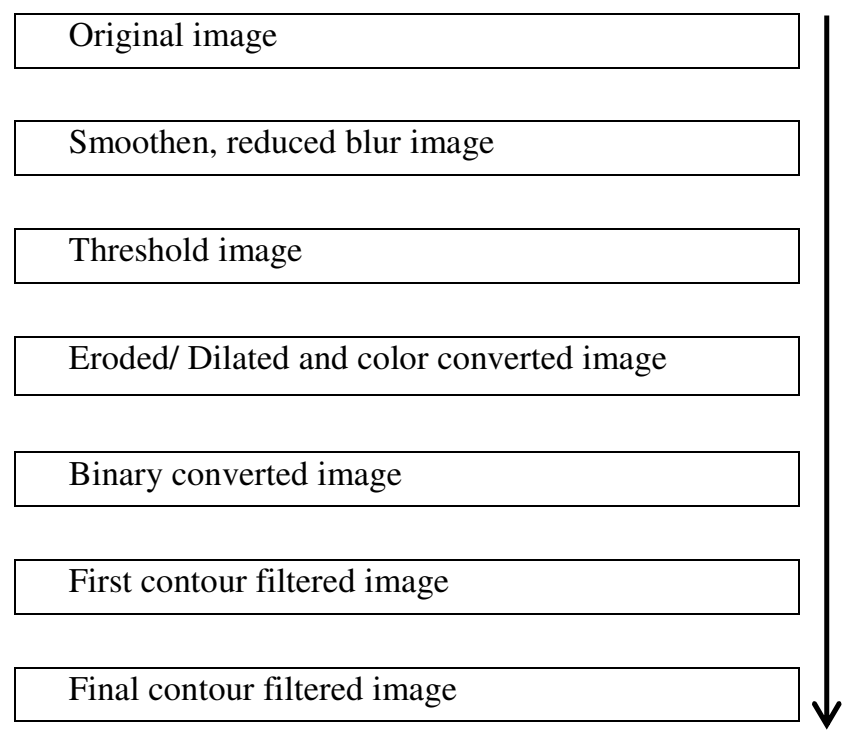

Fig. 2: Image Segmentation Process

Since the requirement was to develop a girth measurement system based on the existing numbering system, QR code/ Bar code based numbering systems were omitted even though these methods could store more information such as date of planting, yield period, etc.

Hence, there were no significant changes done on the existing numbering system with the only change introduced being the use of a stencil to draw the numbers. Empirical data showed that red color provided best threshold characteristics when compared to other commonly used colors including black and blue, thus it was chosen to color the numbers. Further, thin standard strip was introduced to be painted below the numbers and it is used to derive the girth of the tree and will be discussed under section III.

The number identification process is detailed as follows;

First the original image is given as an input to the OpenCV and Visual Basic based image processing system where the first step is to smooth the image to reduce the blur. The next process is to threshold the image in order to remove the background details. Even though OpenCV has an inbuilt function for this purpose, this research deployed a customized threshold function which automatically identifies a threshold value based on image characteristics. After thresholding, the image is color and binary converted to implicitly identify contours. Contours are then drawn for the binary converted images around the dark areas.

In the contour filtration process several filters were used to identify contours belonging to numbers. One such filter will use the contour area.

Contour areas which are not in the range of $400-20000$ are rejected in this filter. After that bounding rectangles are drawn around the detected contour areas.

Bounding rectangles in the algorithm, uses the filtering mechanism based upon the ratio between bounding rectangle area to contour area which must be greater than 1.0. 
Signal \& Image Processing : An International Journal (SIPIJ) Vol.6, No.1, February 2015

This approach was introduced to avoid counting the girth measurement thin strip identifying as a false 1 .

After the filtering processes, a contour class is defined where the contour class contour objects are created with their attributes such as the width, height and midpoint co - ordinates passed at the constructor. These objects are stored in a contour object vector.

There were some algorithms which were attempted prior establishing the optimum contour filtration method. One such algorithm is midpoint algorithm, which assumes that the tree is placed absolutely along the midpoint $x$ value of the image. Hence it is also assumed that the midpoint $\mathrm{x}$ and midpoint $\mathrm{y}$ of the image is also placed at the tree trunk and there is a definite digit. Other digits are found out by checking along a line horizontally and vertically. Requirement to find other bounding rectangles are to have the same height of the bounding rectangle found we first found out. After filtering according to above requirement number detection was not successful up to the level expected. The main reason is that tree is generally not going to be placed at the absolute middle and at the midpoint co - ordinate does not always get an absolute digit.

\section{Number Identification}

One important aspect to consider in the image processing sub system was to identify the digital digits and convert them into numbers. For this purpose, firstly, contour object vector was fed into the popular open source Tessaract optical character recognition engine better known as Tessaract OCR. Even though generic Tessaract algorithm is useful in identification of standard characters, it was proven to be less accurate with the hand written numbers on the Rubber trees and provided erroneous results.

Hence, it was required to develop a unique OCR algorithm based on KNN (K- Nearest Neighbor) [4] classifier method where the engine is trained for recognition of hand written numbers through train data.

This system required larger number set to be processed to correctly identify optical characters. i.e. 100 samples were required per each digit to identify each of the digits $0-9.50$ of those samples are used as test data and other 50 as train data. Preprocessing is done with these 100 samples along with user input data.

Advantage of using KNN algorithm is that it can be successfully programmed and understood than other OCRs. Also if there's no majority agreement it has the explicit rejection option. But at the same time this OCR is sensitive to noise and irrelevant features. Also the memory requirement is higher as it needs sample data as trained data and test data.

\section{Width Measurement}

Successful identification of the tree ID (number) leads to the next step, measurement of tree width. For image processing based width measurement, we have introduced a semicircular thick strip drawn under the tree id and above standard $120 \mathrm{~cm}$ from the ground. 
Signal \& Image Processing : An International Journal (SIPIJ) Vol.6, No.1, February 2015

Even though there are several approaches for segmenting the tree from background, ultimately most successful method was the contour based segmentation with some modifications.

This section shall firstly discuss modified contour based segmentation approach which was used for width measurement and the details of the alternative approaches which we attempted.

\subsection{Contour Based Segmentation and Width Calculation}

During the process of number identification, the final step in contour filtration would have few contours including numbers, width indicating thick strip and few other random contours.

In order to isolate the width indicating thick strip, we have used two logical filters, one being the horizontal width to vertical width ratio greater than one and the other being area of the contour greater than 300 square millimeter. With this approach it was possible to filter out numerical contours and random contours. A bounding rectangle drawn around this colored contour will be used for width measurement.

Even though the above contour may have a curved shape, the linear distance between the $\mathrm{x}$ axis end points would result the width of the tree.

\subsection{Alternate Image Processing Methods for Width Measurement}

\section{a. Blob Detection [5]}

Blob detection is a method to segment an object from its background by Morphological Operations (gradient) and Thresholding. However, this method was not successful because tree could not be uniquely identified as one big blob due to various edges present in the tree trunks and hence provided multiple smaller blobs.

\section{b. Color Based Contour Extraction}

Assuming that the tree will contain the midpoint of the image, the algorithm was firstly developed to examine significant pixel differences from the midpoint in horizontal directions.

The algorithm was further developed to examine pixels vertically from the midpoint in order to learn the pixel characteristics of the tree trunk and then processed in horizontal directions for segmenting of the tree.

However, this method was not successful due to the diverse pixel characteristics of the tree trunk.

c. Global and Local Thresholding

In global thresholding, the image was segmented by differentiating the pixels by a certain pixel threshold value. Since this method provided poor results, we attempted the Local thresholding by dynamically allocating different thresholding values for $128 \mathrm{X} 128$ pixel regions. The local threshold value was obtained based on two pixel characteristics, mean intensity and standard deviation of the intensity. By allocating 128X128 regions, it was ensured that the neighborhood 
Signal \& Image Processing : An International Journal (SIPIJ) Vol.6, No.1, February 2015

chosen was large enough to cover sufficient foreground and background pixels, to avoid a poor threshold value being calculated

\section{d. Grab Cut Function}

Grab cut function was developed based on Graph cut method by assuming the pixel points are Cartesian coordinates of a 2D graph. In this iterative method to differentiate foreground and background, it was required to define a minimum cut containing source and sink as per the Ford $\&$ Fulkerson theorem $[6,8]$. Assuming that the centre point of the tree would contain the tree trunk, a rectangle was defined. Further, Grab cut algorithm [7] was further developed to automatically initialize the segmentation process to suit the research requirement.

\subsection{Statistical Derivation of Perimeter}

Even though it was possible to successfully measure the width of a Rubber tree from above Image Processing methods, the derivation of perimeter from the standard equation (1) was not straight forward considering the irregularities of the tree trunk.

$$
\mathrm{P}=\Pi \text {. D }
$$

Hence, it was required to define a statistical $\mathrm{K}$ value for deriving perimeter from tree width or diameter. However, it was observed that although young Rubber trees kept a cylindrical shape, the aged Rubber trees had increased irregularities. Considering above factor, it was required to define $\mathrm{K}$ values for width class. Ultimately, the final $\mathrm{K}$ values were defined after statistical analysis of actual perimeter and image processing perimeter for thousand samples covering all the width classes.

$$
\mathrm{P}=\mathrm{K} . \text { П. D }
$$

\section{B. GUI and Database}

A graphical user interface (GUI) was developed based on Microsoft Foundation Classes (MFC) and $\mathrm{C}++$ language, which provided an interface to store the measured data in a SQL based database system.

\section{Design of Hardware Module}

The requirement of the hardware module was to capture a good quality image and transfer it to the image processing sub system which will then calculate the width and derive tree identity. Further to that, the Grab cut function used in the image processing system required to have a minimum 5 mega pixel $(2,592 \times 1,944)$ resolution and approximately 70 percent of the image contained tree trunk for efficient segmentation. 
Signal \& Image Processing : An International Journal (SIPIJ) Vol.6, No.1, February 2015

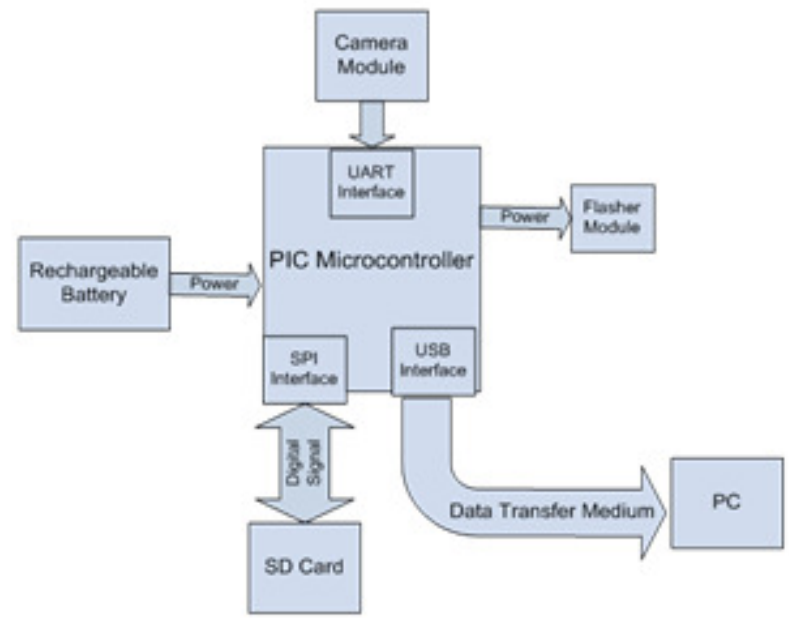

Fig. 3: Hardware Architecture

Phase 1

During the phase one, development of the total hardware module including the camera module was considered from the scratch. But, it was proven that a compact camera module would be best suited for core requirements.

Phase 2

\section{Derivation of focal length}

In order to facilitate with segmenting process, it was required to have a better field of view which would help in containing tree trunk in a larger percentage of the image. The different focal lengths were compared with field of view based on equation 3 where $f$ stands for focal length. It was required to obtain a better field of view compared to human eye of 50 degrees.

$$
\text { Field of view }=2 * \tan ^{-1}\left(\frac{3.08}{f}\right)
$$

2. Derivation of minimum and optimum distance from the tree

As per, field tested samples, the maximum width of a rubber tree was $19.5 \mathrm{~cm}$. Hence, it was required to derivate the minimum and optimum distance ( 70 percent of the image containing tree trunk). The statistical experiments were done based on the equation 4 where $\mathrm{p}, \mathrm{f}, \mathrm{d}, \mathrm{w}_{\mathrm{o}}, \mathrm{w}_{\mathrm{s}}$ being percentage of object on image sensor, focal length, distance from object, maximum tree width, sample tree width respectively.

$$
p=h_{0} *\left(\frac{1}{\frac{1}{f}-\frac{1}{d}}\right) / h_{g}
$$

Hardware design was developed based on above calculations where as a permanent metal gauge was introduced on the enclosure to aid in maintaining the optimum length. 
Signal \& Image Processing : An International Journal (SIPIJ) Vol.6, No.1, February 2015

\section{DISCUSSION AND FUTURE WORK}

The immediate requirement for this project was to develop an efficient image processing solution to existing manual girth measurement system without introducing many changes to prevailed mechanisms.

When analyzing the methods which can improve the proposed system, we found that if $1 \mathrm{D}$ bar codes or QR codes are used in the numbering system, it would be possible to store much data about the plantation. Further, these 1D bar codes/ QR codes can be automatically generated on annual basis based on the iterative information store in the management database system. We would highly recommend a non-invasive methods such as fixing a standard number plate on the tree using an elastic strip where above mentioned 1D bar codes/ QR codes can be inserted to these number plates on annual basis. This will help in storing critical information such as growth history, fertilizer requirements, and yield production on the number plate itself. Since QR codes can include error correcting algorithms, the data can be restored even when the image is affected by fungus and other artifacts up to 30 percent.

\section{A. Binary 2D Barcode}

Considering the information requirement on the field and decoding flexibility, we have developed a binary 2D barcode system which can store moderate level of information including tree id, low yield indication, low early growth indication.

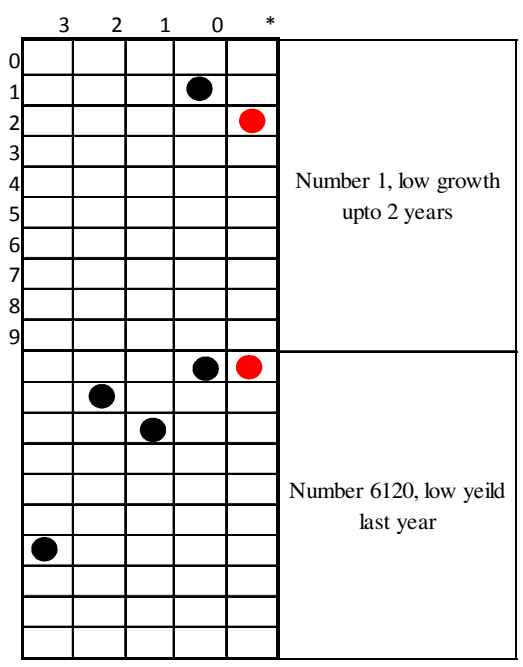

Fig. 4: Binary 2D Bar Code

Figure 4 details a referent 2D bar code system which can be used for above purpose.

\section{RESULTS}

The field testing was carried out in Mahaoya Rubber estate operated by Lalan Plantations. 1000 image samples were tested with the final system and compared with actual girth measurement data. 
Signal \& Image Processing : An International Journal (SIPIJ) Vol.6, No.1, February 2015

As mentioned in the section 3.2, defining the $\mathrm{K}$ value was achieved from above 100 samples. I was observed that even though young Rubber trees maintained the cylindrical shape, aged Rubber trees developed an irregular, ecliptic shape.

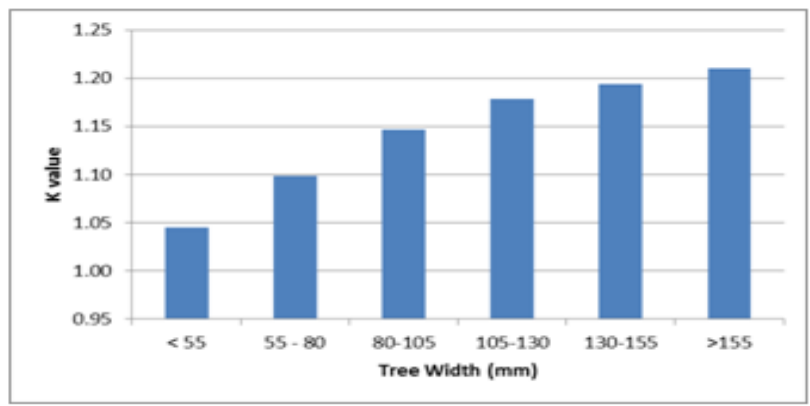

Fig. 5: Derivation of K value ( eq. 2) for tree width classes

During the segmentation process, the most successful segmentation method was contour filtering. Even though customized Grab cut function and color based segmentation provided reasonably better results, the accuracy levels were below the required levels specially for width measurement.

Figure 6 details the contour based segmentation process while figure 7 and figure 8 details the Grab Cut Function and Color Based Segmentation results
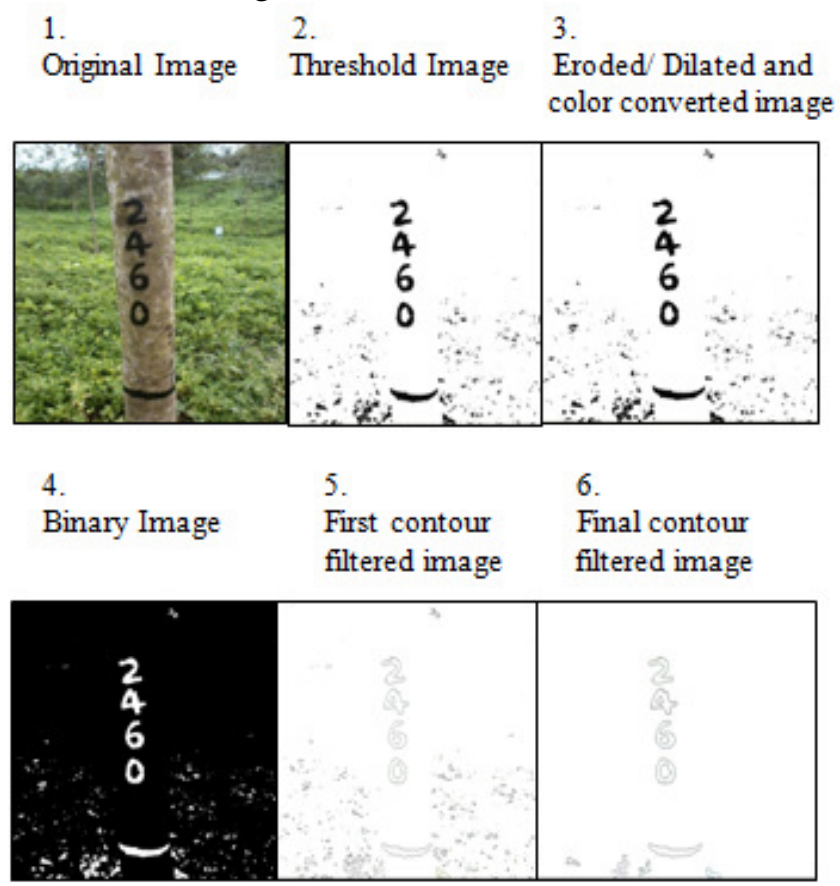

Fig. 6: Number Identification and location steps using contour filtering 
Signal \& Image Processing : An International Journal (SIPIJ) Vol.6, No.1, February 2015

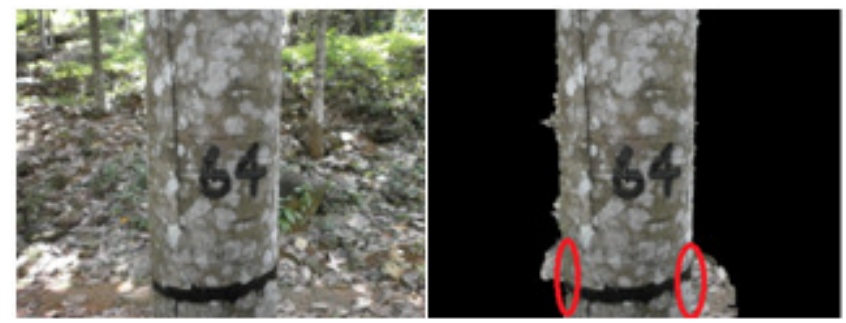

Figure 7: Segmentation based on Grab Cut Function

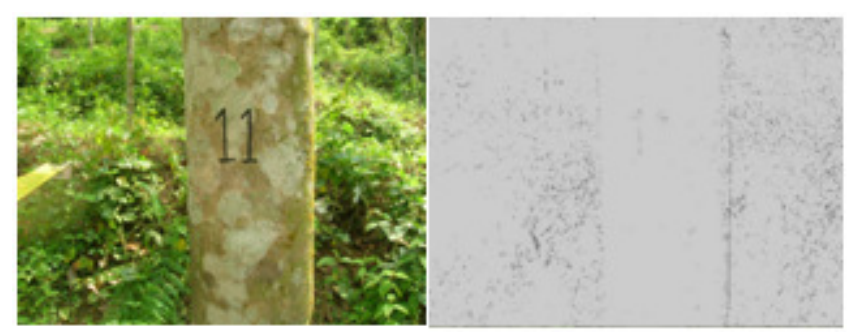

Figure 8 : Color based Edge Detection

The graph in Figure 9 details a sample of measured and actual girth parameters. We have observed $-5<\mathrm{x}<+6 \mathrm{~mm}$ range variations in the measured results. The Mean error and Standard Deviation were $\mathbf{+} \mathbf{3 . 4 7 2} \mathbf{~ m m}$ and $\mathbf{1 . 2 0 9 7 5} \mathbf{~ m m}$ for the total sample respectively.

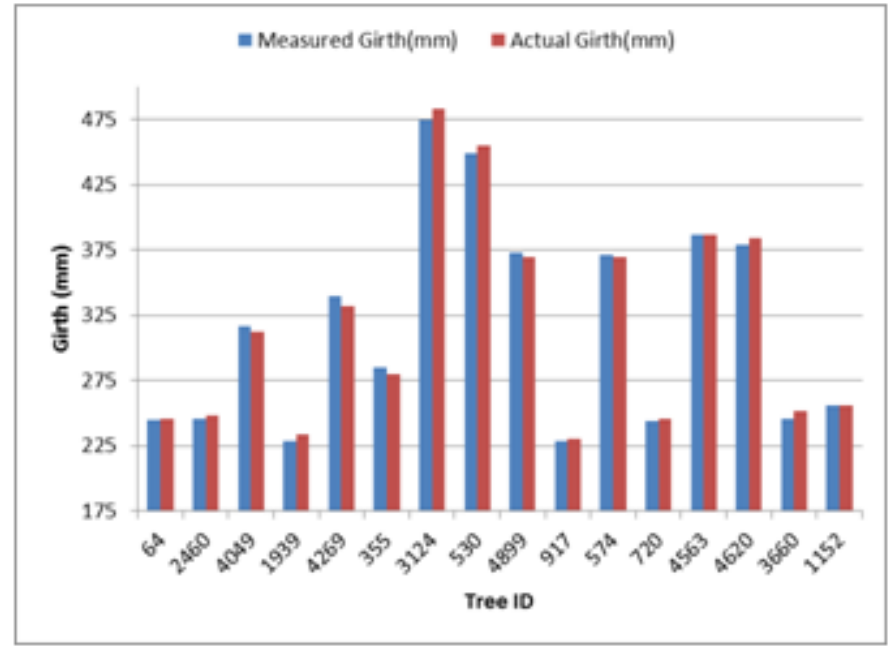

Fig 9: Measured and Actual Girth (Randomly Selected)

\section{CONCLUSION}

As per our research outcomes, it is observed that generic theoretical image processing algorithms need to be developed as per the application requirements and environmental characteristics. The proposed extended KNN algorithm presents such scenario identifying numbers on tree trunk. 
Signal \& Image Processing : An International Journal (SIPIJ) Vol.6, No.1, February 2015

Use of technology in the traditional fields such as plantations would definitely benefit in the future. In order to maximize the benefit it is required to combine different technology and expertise to develop intelligent systems to deliver total solutions fulfilling practical requirements in the industry. Considering on rubber industry, above system can be further developed by integrating functionalities such as weather measurement, texture analysis and soil quality measurement.

\section{ACKNOWLEDGMENT}

We would like to extend our gratitude to Prof. Asoka Nugawela of Lalan Plantations for the invaluable feedback and enthusiastic engagement with the research.

\section{REFERENCES}

[1] Gonçalves P., Scaloppi E, Martins M, Moreno R., Branco R, Gonçalves E, (2011, November), Assessment of growth and yield performance of rubber tree clones of the IAC 500 series, Pesq. agropec. bras., Brasília, v.46, n.12, p.1643-1649, dez. 2011[online]

[2] Learning OpenCV: Computer Vision with the OpenCV Library by Gary Bradski

[3] OpenCV 2 Computer Vision Application Programming Cookbook by Robert Laganière

[4] Prasad P., Nagarjuna D., Ali N., (2009), k Nearest Neighbours Algorithm, Academia.edu [online]

[5] Lu S.,Tsechpenakis G. and Metaxas D., Blob Analysis of the Head and Hands: A Method for Deception Detection, [online]

[6] Graph Cuts in vision \& graphics: Theories \& Application Boykov October 22, 2007

[7] Efficient graphcuts for unsupervised image segmentation using probabilistic sampling and SVD based approximation - Keuchel Jens; Schnorr Christoph; January 23, 2008

[8] L.H. Harper, J.D. Chavez, (2002 April 30). Duality Theorems for a Continuous Analog of FordFulkerson Flows in Network [online]

[9] Yadraj Meena , Dr. Ajay Mittal, (2002, July), Blobs and Cracks Detection on Plain Ceramic Tile Surface, International Journal of Advanced Research in Computer Science and Software Engineering, [online], Volume 3, Issue 7a 